\title{
Kaisa Sere: In Memoriam
}

\author{
Luigia Petre, Elena Troubitsyna, and Marina Waldén \\ Department of Information Technologies, Åbo Akademi University, Joukahaisenkatu 3-5A, 20520, Turku, Finland
}

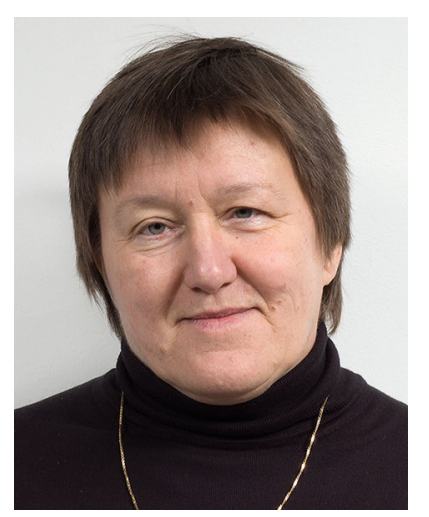

Kaisa Sere, a wonderful person and scientist, passed away on December 5, 2012, after a long and devastating illness. Apart from her husband Thorbjörn Andersson and the rest of her family, Kaisa is going to be missed by so many colleagues in Finland and worldwide.

Kaisa was born in 1954 in Kokkola, Finland. She graduated from Åbo Akademi University with a M.Sc. in Mathematics and received a Ph.D. in Computer Science from the same university in 1990. She was a lecturer at Ohio State University, US, during 1984-1985 and a post-doctoral researcher in the Department of Computer Science, at Utrecht University, the Netherlands, during 1991-1992. She held an Associate Professorship at the University of Kuopio, in the Department of Computer Science and Applied Mathematics, during 1993-1998, she became a Docent of Computer Science at the same department in 1997, and held a senior research professorship from the Academy of Finland during 1998-1999. She became a full

professor of Computer Science and Engineering at Åbo Akademi University on August 1st, 1998. During 20102011, she also held a senior researcher position from the Academy of Finland and a vice-rectorship at Åbo Akademi University.

One of the major contributions of Kaisa Sere's career was made to the refinement research. In her Ph.D. thesis Stepwise Derivation of Parallel Algorithms [Sere90] she developed a refinement methodology for deriving provably correct parallel and distributed algorithms with Ralph-Johan Back as her supervisor. As a formal framework for her research she used Action Systems by Back and Kurki-Suonio [BaKu83]. Within that framework she then developed with Back a superposition refinement method [BaSe96a] that is a powerful variant of the general form of refinement for increasing the degree of parallelism in systems. She was also involved in exploring how this superposition method could be used more generally, as a tool for structuring the verification process as well as the algorithm, when applying it backwards [SeWa96, SeWa01]. In order to have tool support for superposition refinement within the Action Systems framework, she initiated research on how the tool support for Jean-Raymond Abrial's B-Method [Abr96] could be used for the Action Systems development [WaSe98], which resulted in the B Action Systems framework. This research had later an influence on the development of Event-B by Abrial [Abr10] and on its tool support, the Rodin platform [ABHHMV, Rodin]. As she was keen on investigating new application areas of formal methods, she also inspired a line of research on how to develop Systems-on-Chip [TsSeP110] and Network-on-Chip architectures, using the B Action Systems and later Event-B, with their tool support.

The development of parallel and distributed systems as well as their performance were always among Kaisa Sere's prime research interest; she aimed at achieving a high degree of parallelism in distributed algorithms. She investigated with Back how Action Systems could be used for developing modular systems, where the modules have autonomous internal activity and communicate via, for example, shared variables or remote procedure calls [BaSe96b]. As a consequence, they also extended the Action Systems formalism with procedures and proposed

Correspondence and offprint requests to: L. Petre, E-mail: luigia.petre@abo.fi 
proof rules for developing and reasoning about their correctness [SeWa00]. Via atomicity refinement of remote procedures the degree of parallelism was even further increased. Together with Emil Sekerinski she extended the modular ideas and created a theory of prioritising composition of processes [SeSe96], where the processes were described as action systems in which one process may have priority over another. Moreover, she developed the modular ideas for Action Systems further by employing a method for synthesizing components from a high-level specification via correctness-preserving transformation steps [PSW04]. Tool support for the method was provided via the B-Method and the B Action Systems. In this research, she also promoted different scheduling mechanisms for Action Systems. During her last years, she was involved in further developing the ideas of introducing explicit control flow also in the Event-B framework [BDSW12].

Contemporary computing paradigms provided a constant theme and inspiration for Kaisa Sere's research, which was often aimed at proposing design methods for systems following these paradigms. Based on the flexible Action Systems framework, she addressed object-orientation, mobility, coordination, peer-to-peer and wireless networks, Network-on-Chip architectures and context-awareness. The extensions to Action Systems that were needed in order to accommodate these paradigms were conservative, meaning that they were semantically explained in the basic framework. Thus, the refinement methodology could be reused to develop systems based on all these paradigms. By addressing object-orientation with the Action Systems framework, stepwise refinement was demonstrated for developing object-oriented systems, while Actions Systems gained the notion of identity: objects could communicate only if they knew each other's identity [BoKoSe98, BoKoSe99]. By addressing mobility with the Action Systems framework, mobile systems were endowed with the stepwise refinement methodology, while Action Systems gained the notion of location guard: a system could execute only when all the necessary resources were within its location-based cell [PeSeWa99]. It is noteworthy that object-orientation and mobility simply constrain the otherwise non-deterministic execution model of Action Systems, as does prioritizing composition [SeSe96] introduced earlier. These three paradigms were also combined to result in coordinating mobile objects [PeSe99], i.e., developing object-oriented systems where some classes produced mobile objects and some classes produced coordinator objects, so that some mobile objects could be coordinated by specific coordinator objects while others were not. In addition, modelling context-awareness with the Action Systems framework provided yet another methodology to study module interdependencies and to restrict non-determinism [NSYS06, NeSe09].

Stepwise refinement was also proposed for developing wireless [KLPS10], peer-to-peer [YaSe00] and Networkon-Chip [KPSD12, PSTLP11] architectures in Event-B, based on tool support from the Rodin platform. Correctness properties were automatically proved for these systems so that they were kept valid during the stepwise development. Interestingly, for macro-level networks (wireless and peer-to-peer networks), when continuing the stepwise development with distributing the specification, rules valid for Action System development were rediscovered [PeSaSe12, KLPS13] for Event-B, thus validating Kaisa Sere's earlier research [BaSe96a, WaSe98] in the context of automatic theorem provers.

The design method for all these paradigms typically offered an iterative, refinement-based approach referred to as correctness-by-construction; however, usability and reuse played an increasingly important role in Kaisa Sere's research. With respect to usability, she studied non-functional properties such as energy-awareness [PeSe10], network availability [PeSeWa06, PeSeWa11] and trustworthiness [NeSe13]; she also started a project on how to improve proofs in an automated theorem prover [DLPSS12]. At an earlier stage, integration of object-oriented Action Systems with the industrially-relevant modelling language UML demonstrated Kaisa Sere's interest in offering a visual interface to formal modelling [PeSe00]. With respect to reuse, she studied inheritance in objectorientation [BoKoSe98, BoKoSe99] as well as design and refinement patterns in wireless networks [KLPS12], proposing a formal network theory to address not only macro-level network development but Network-on-Chip systems as well [KaPeSe13]. The vision was that, similarly to software developers using predefined libraries, we could have libraries of proved (network) models. By developing these models via refinement, one would get a correct-by-construction reuse of correct models.

Kaisa Sere was a scholar who was prolific in both theoretical and applied research. She was one of the main drivers behind a succession of remarkable EU projects in the field of formal methods. These projects have significantly advanced the state-of-the-art in formal methods and refinement in particular. In the EU project MATISSE: Methodologies and technologies for industrial strength systems engineering (EU Fifth Framework IST Programme, 2000-2003) [MATISSE] the focus was put on strengthening state-based modelling to address such issues as modelling time and distributed behavior, integration with graphical modelling techniques and safety analysis. Kaisa Sere's group was cooperating closely with Perkin Elmer Life Sciences: together with engineers from the company, they studied the use of formal techniques in the development of highly reliable systems for drug 
discovery - the liquid handling workstation Fillwell ${ }^{\mathrm{TM}}$. The project has clearly demonstrated that, to warrant adoption of formal techniques in industrial practice, we need to develop powerful yet open and extendable tools supporting refinement-based development.

The EU project RODIN: Rigorous Open Platform for Complex Systems (EU Sixth Framework IST Programme, 2004-2007) [RODIN] has resulted in achieving the above goal. The main outcome of the project is the Rodin platform - an industrial-strength automated environment for modelling and refinement in Event-B. In the RODIN project, significant advances were made in creating powerful techniques for the development of dependable systems from various industrial domains. Kaisa Sere's group was cooperating closely with Nokia Research Center, strengthening by formal modelling an industrial service-oriented approach to developing communicating systems and communication protocols and augmenting this approach with fault tolerance mechanisms. The RODIN project resulted in creating a large collection of reusable modelling and refinement patterns for developing dependable systems as well as in powerful tool support to automate the development.

The next logical step was an integration of the created methods and tools in industrial practice. This was the focus of the EU integrated project DEPLOY: Industrial deployment of advanced system engineering methods for high productivity and dependability (EU Seventh Framework IST Programme, 2008-2012) [DEPLOY]. In this project, the development of the refinement theory and its automated tool support was driven by industrial needs. The leading European companies from automotive, railway, aerospace and business information systems (Bosch, Siemens Transportation, Space Systems Finland and SAP) joined forces to find industry-specific solutions for integrating formal modelling into their development processes. The project had provided the Rodin platform with the industrial stress-test that resulted in its further strengthening and extension.

Thus, 12 years of intensive research in EU projects made many of Kaisa Sere's aspirations come true. Formal techniques were strengthened and extended to cover novel computational phenomena; the Rodin platform provided the refinement approach with industrial-strength automated support, used worldwide; and the formal techniques were used to address the challenges of real-life industrial systems. These remarkable achievements were fundamentally influenced by Kaisa Sere's research and visions.

Besides these major EU-projects, Kaisa Sere won an impressive number of other research grants: DESIRE: Design of reliable embedded systems (Academy of Finland, 1999-2001); Asynchronous design of DSP Architectures (Academy of Finland, 2000-2002); served in the ACiD-WG: Asynchronous Circuit Design Working Group (EU Fifth Framework IST Programme, 2000-2004); FOSSE: Formal Systems and Software Engineering (Academy of Finland, 2001-2004); research group leader and the vice-leader of the Academy of Finland Centre of Excellence on Formal Methods in Programming (2002-2007); research leader in the project ITCEE: Improving Transient Control and Energy Efficiency by digital hydraulics (Tekes-Kitara programme, 2005-2008); NODES: Network of Dependable Systems (Nordforsk, 2007-2012); responsible site leader of the projects EFFIMA/DiHy: Digital Microhydraulics (2009-2014) and EFFIMA/Digihybrid: Regenerative hydraulic hybrid with digi-valve and multi chamber cylinder technology (2011-2014) (the Fimecc Strategic Centre for Technology and Innovation in cooperation with TEKES); task leader in the project RECOMP: Reduced certification costs for trusted multicore platforms (Artemis JTI in the EU 7th framework programme, 2010-2013); FResCo: High-quality Measurement Infrastructure for Future Resilient Control Systems (Academy of Finland, 2013-2015).

Kaisa Sere was invited to participate in numerous boards and committees of a scientific, administrative and evaluation nature. In 2007, she was a member of the committee to evaluate the NOKUT engineering programmes in Norway. During 2006-2009 she was a member of the TUTOR group for coordinating research programs at the Academy of Finland. During 2004-2009 she was a member of the ESF Standing Committee for Physical and Engineering Sciences (PESC) and during 2007-2009 also a member of the ESF core group for PESC. She was the vice-chair and a member of the Research Council for Natural Sciences and Engineering at the Academy of Finland during 2004-2009. Since 2008 she was the Finnish representative of the Governing Board of the ARTEMIS Joint Undertaking Initiative in Embedded Computing Systems. She was a member of evaluation committees for Ph.D. thesis evaluations (12 appointments), professorship competence (14 appointments), and docentships ( 3 appointments). She has been organizing and chairing several major conferences in her field of research of Formal Methods.

Kaisa Sere has supervised 19 Ph.D. students: Fredrik Degerlund: Scheduling of Guarded Command Based Models, from Åbo Akademi University, 2012 (jointly with Marina Waldén); Mats Neovius: Trustworthy Context Dependency in Ubiquitous Systems, from Åbo Akademi University, 2012 (jointly with Luigia Petre); Marta Olszewska: On the Impact of Rigorous Approaches on the Quality of Development, from Åbo Akademi University, 2011 (jointly with Marina Waldén); Maija Marttila-Kontio: Visual Data Flow Programming Languages, from University of Eastern Finland, 2011; Leonidas Tsiopoulos: Formal Model-Based Development of Network-on-Chip 
Systems, from Åbo Akademi University, 2010; Pontus Boström: Formal specification and verification of systems using model specific languages, from Åbo Akademi University, 2008 (jointly with Marina Waldén); Tomi Westerlund: Time aware modeling and analysis of Systems-on-Chip, from University of Turku, 2008 (jointly with Juha Plosila); Dubravka Ilic: Formal reasoning about dependability in model-driven development, from Åbo Akademi University, 2007 (jointly with Elena Troubitsyna); Orieta Celiku: Mechanized reasoning for dually nondeterministic and probabilistic programs, from Åbo Akademi University, 2006 (only in the final phase, jointly with Annabelle McIver and Joakim von Wright); Mehran Gomari: On the generalisation Ability of Bayesian Neural Networks, from the University of Turku, 2005 (jointly with Timo Järvi); Lu Yan: Systematic design of ubiquitous systems, from Åbo Akademi University, 2005; Luigia Petre: Modeling with Action Systems, from Åbo Akademi University, 2005 (jointly with Ralph-Johan Back); Rimvydas Rukšènas: Formal concurrent components, from Åbo Akademi University, 2004; Mauno Rönkkö: Stepwise Development of Hybrid Systems, from Åbo Akademi University, 2001, awarded the Best Computer Science thesis in Finland in 2001 (jointly with Anders P. Ravn); Tiberiu Seceleanu: Systematic design of synchronous digital circuits, from Åbo Akademi University, 2001; Elena Troubitsyna: Stepwise development of dependable systems, from Åbo Akademi University, 2000; Virpi Kasurinen: Informal and formal requirements specification, from University of Kuopio, 1999; Juha Plosila: Self-timed circuit design — the action systems approach, from University of Turku, 1999; Marina Waldén: Formal reasoning about distributed algorithms, from Åbo Akademi University, 1998 (jointly with Ralph-Johan Back). At the moment of her passing away, seven Ph.D. students still had her as a (co-)supervisor.

The flagship of Kaisa Sere's career was her passion for research. As can be seen from the above sketch of her biography, she has been extremely successful in attracting research funding, from the most competitive scientific forums such as the Academy of Finland and the European Commission. This research funding enabled her to develop her own research group: the Distributed Systems Laboratory. At the time of her passing away, this group comprised 20 researchers: 4 senior researchers, 6 post doctoral researchers, $8 \mathrm{Ph}$.D. students and $2 \mathrm{M}$.Sc. students. During fall 2012, there were 9 applications submitted to the Academy of Finland from the Distributed Systems laboratory, one being Kaisa Sere's own application. In January 2013, two European Commission applications were submitted from the same laboratory. Her research success and profound impact on the computer science field is also demonstrated in her publication record. According to the Publish or Perish software, in December 2012 she had 154 articles, 1,953 citations, an h-index of 23, and an average of 78 cites per year during a career of 25 years. If one checks these numbers now, they are already different: she had so much ongoing research at the time she fell ill, that papers bearing her name keep getting published. This shows how much involved she was until the very end.

\section{References}

[Abr96] Abrial J-R (1996) The B book: assigning programs to meanings. Cambridge University Press

[Abr10] Abrial J-R (2010) Modelling in event-B: systems and software engineering. Cambridge University Press

[ABHHMV] Abrial J-R, Butler M, Hallerstede S, Hoang TS, Mehta F, Voisin L (2010) Rodin: an open toolset for modelling and reasoning in event-B. Int J Softw Tools Technol Transf 6:447-466

[BaKu83] Back R-JR, Kurki-Suonio R (1983) Decentralization of process nets with centralized control. In: Proceedings of the 2nd ACM SIGACT-SIGOPS symposium of principles of distributed computing, pp 131-142

[BaSe96a] Back R-JR, Sere K (1996) Superposition refinement of reactive systems. Formal Aspects Comput 8(3):324-346

[BaSe96b] Back R-JR, Sere K (1996) From action systems to modular systems. Softw Concepts Tools (17):26-39

[BoKoSe98] Bonsangue M, Kok JN, Sere K (1998) An approach to object-orientation in action systems. In: Jeuring J (ed) Proceedings of MPC'98-fourth international conference on mathematics of program construction. Lecture notes in computer science, vol. 1422. Springer, pp $68-95$

[BoKoSe99] Bonsangue M, Kok JN, Sere K (1999) Developing object-based distributed systems. In: Ciancarini P, Fantechi A, Gorrieri R (eds) Proceedings of FMOODS'99 - third IFIP international conference on formal methods for open object-based distributed systems. IFIP the international federation for information processing. Springer science+business media, vol 10. New York, pp 19-34

[BDSW12] Boström P, Degerlund F, Sere K, Waldén M (2012) Derivation of concurrent programs by stepwise scheduling of Event-B models. Formal Aspects Comput doi:10.1007/s00165-012-0260-5

[DEPLOY] DEPLOY (2012) Industrial deployment of advanced system engineering methods for high productivity and dependability. EU Seventh Framework IST Programme, 2008-2012. http://www.deploy-project.eu/

[DLPSS12] Diaconescu D, Leustean I, Petre L, Sere K, Stefanescu G (2012) Refinement-preserving translation from event-B to registervoice interactive systems. In: Latella D, Treharne H (eds) 9th International conference on integrated formal methods (iFM 2012). Lecture notes in computer science. vol 7321. Springer, pp 221-236 
[KLPS10] Kamali M, Laibinis L, Petre L, Sere K (2010) Self-recovering sensor-actor networks. In: Mousavi M, Salaün G(eds) Proceedings of the 9th international workshop on the foundations of coordination languages and software architectures (FOCLASA 2010). Electronic proceedings in theoretical computer science (EPTCS) vol 30. pp 47-61

[KLPS12] Kamali M, Laibinis L, Petre L, Sere K (2012) Formal development of wireless sensor-actor networks. Sci Comput Progr J (SCP). doi:10.1016/j.scico.2012.03.002 (In press)

[KLPS13] Kamali M, Laibinis L, Petre L, Sere K (2013) A distributed design of a network recovery algorithm. Int J Crit Comput Based Syst 4(1):45-68 (Inderscience Publishers)

[KaPeSe13] Kamali M, Petre L, Sere K (2013) NetCorre: a hierarchical framework and theory for network design. (Submitted April 2013)

[KPSD12] Kamali M, Petre L, Sere K, Daneshtalab M (2012) Refinement-based modeling of 3D NoCs. In: Arbab F, Sirjani M (eds) 4th IPM international conference on fundamentals of software engineering (FSEN). Lecture Notes in Computer Science, vol 7141. Springer, pp 236-252

[MATISSE] MATISSE (2003) Methodologies and technologies for industrial strength systems engineering. In: EU fifth framework IST programme, 2000-2003. http://cordis.europa.eu/result/report/rcn/30461 en.html

[NeSe09] Neovius M, Sere K (2009) Formal modular modelling of context-awareness. In: de Boer FS, Bonsangue MM, Madelain E (eds) Formal methods for components and objects, 7th international symposium, FMCO 2008, revised lectures. Lecture notes in computer science, vol 5751.pp 102-118

[NeSe13] Neovius M, Sere K (2013) Mastering the relevance of subjective information in ubiquitous computing. J Commun Inform Sci AICIT 3(4):27-44

[NSYS06] Neovius M, Sere K, Yan L, Satpathy M (2006) A formal model of context-awareness and context-dependency. In: Hung Dang V, Paritosh P (eds) Proceedings of the fourth IEEE international conference on software engineering and formal methods (SEFM'06). IEEE computer society press, pp 177-185

[PeSe99] Petre L, Sere K (1999) Coordination among mobile objects. In: Ciancarini P, Wolf A (eds) Proceedings of COORDINATION'99- the $3^{\text {rd }}$ international conference on coordination languages and models. Lectures notes in computer science, vol 1594. Springer, pp 227-242

[PeSe00] Petre L, Sere K (2000) Developing control system components. In: Grieskamp W, Santen T, Stoddart B (eds) Proceedings of IFM'2000 - The $2^{\text {nd }}$ international conference on integrated formal methods. Lecture notes in computer science, vol 1945. Springer, pp 156-175

[PeSe10] Petre L, Sere K (2010) Towards sustainable development of energy-aware systems. Int J Embed Real Time Commun Syst IGI Global 1(2):45-60

[PeSaSe12] Petre L, Sandvik P, Sere K (2012) Node Coordination in Peer-to-Peer Networks. In: Sirjani M (ed) COORDINATION 2012. Lecture Notes in Computer Science, Springer-Verlag 7274:196-211

[PSTLP11] Petre L, Sere K, Tsiopoulos L, Liljeberg P, Plosila J (2011) Towards self-placing applications on 2D and 3D NoCs. In: CongVinh P (ed) Autonomic networking-on-chip: bio-inspired specification, development, and verification, embedded multi-core systems (EMS) Book Series. CRC Press, pp 165-187

[PeSeWa99] Petre L, Sere K, Waldén M (1999) A Topological Approach to Distributed Computing, G. Ştefănescu (ed) Proceedings of FCT'99 - Workshop on Distributed Systems, Electronic Notes in Theoretical Computer Science. Elsevier Science 28:97-118

[PeSeWa06] Petre L, Sere K, Waldén M (2006) A Language for modeling network availability. In: Liu Z, He J (eds) Proceedings of the 8th international conference on formal engineering methods (ICFEM 2006). Lecture notes in computer science. vol 4260. Springer, pp 639-659

[PeSeWa11] Petre L, Sere K, Waldén M (2011) Network availability for distributed applications. In: Petre L, Sere K, Troubitsyna E (eds) Dependability and computer engineering: concepts for software-intensive systems. IGI Global, pp 36-57

[PSW04] Plosila J, Sere K, Waldén M (2005) Asynchronous system synthesis. Sci Comput Program 55:259-288

[RODIN] RODIN (2007) Rigorous open platform for complex systems. In: EU sixth framework IST programme, $2004-2007$. http://rodin.cs.ncl.ac.uk/

[Rodin] Rodin tool platform (2013) http://www.event-b.org/platform.html. Accessed 21 June 2013

[SaSe11] Sandvik P, Sere K (2011) Formal analysis and verification of peer-to-peer node behaviour. In: Liotta A, Antonopoulos N, Di Fatta G, Hara T, Hieu Vu Q (eds) The third international conference on advances in P2P Systems (AP2PS 2011). IARIA, pp $47-52$

[Sere90] Sere K (1990) Stepwise derivation of parallel algorithms. Ph.D. thesis, Åbo Akademi University

[SeSe96] Sekerinski E, Sere K (1996) A theory of prioritizing composition. Comput J 39(8):701-712

[SeWa96] Sere K, Waldén M (1996) Reverse engineering distributed algorithms. J Softw Maint Res Pract 8(2):117-144

[SeWa00 Sere K, Waldén M (2000) Data refinement of remote procedures. Formal Aspects Comput 12(4):278-297

[SeWa01] Sere K, Waldén M (2001) Structuring and verifying distributed algorithms. Nordic J Comput 8(2):193-218

[TsSeP110] Tsiopoulos L, Sere K, Plosila J (2010) Modeling communication in multi-processor systems-on-chip using modular connectors. Int J Embed Real Time Commun Syst 1(2):23-44

[WaSe98] Waldén M, Sere K (1998) Reasoning about action systems using the B-Method. Formal Methods Syst Design 13(1):5-35

[YaSe00] Yan L, Sere K (2003) Stepwise development of peer-to-peer systems. In: 6th international workshop in formal methods (IWFM'03) British computer society (BCS), eWiC - electronic workshops in computing, pp 1-16

Published online 9 January 2014 